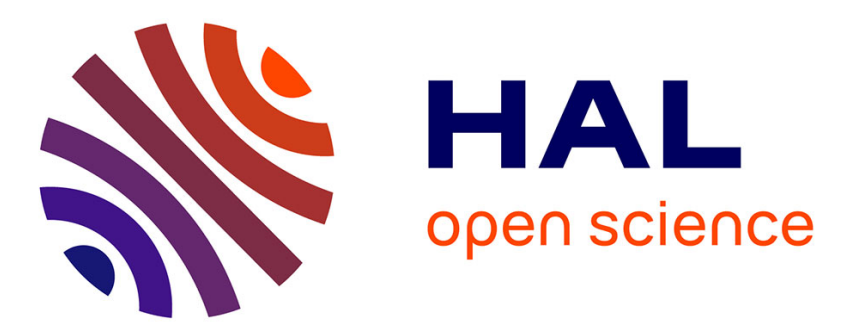

\title{
Prediction bands for solar energy: New short-term time series forecasting techniques
}

Michel Fliess, Cédric Join, Cyril Voyant

\section{To cite this version:}

Michel Fliess, Cédric Join, Cyril Voyant. Prediction bands for solar energy: New short-term time series forecasting techniques. Solar Energy, 2018, 166, pp.519-528. 10.1016/j.solener.2018.03.049 . hal-01736518

\section{HAL Id: hal-01736518 \\ https://hal-polytechnique.archives-ouvertes.fr/hal-01736518}

Submitted on 17 Mar 2018

HAL is a multi-disciplinary open access archive for the deposit and dissemination of scientific research documents, whether they are published or not. The documents may come from teaching and research institutions in France or abroad, or from public or private research centers.
L'archive ouverte pluridisciplinaire HAL, est destinée au dépôt et à la diffusion de documents scientifiques de niveau recherche, publiés ou non, émanant des établissements d'enseignement et de recherche français ou étrangers, des laboratoires publics ou privés. 


\title{
Prediction bands for solar energy: New short-term time series forecasting techniques
}

\author{
Michel Fliess ${ }^{\mathrm{a}, \mathrm{d}, *}$, Cédric Join ${ }^{\mathrm{b}, \mathrm{d}, \mathrm{e}}$, Cyril Voyantc ${ }^{\mathrm{c}, \mathrm{f}}$ \\ ${ }^{a}$ LIX (CNRS, UMR 7161), École polytechnique, 91128 Palaiseau, France. \\ Michel.Fliess@polytechnique.edu \\ ${ }^{b}$ CRAN (CNRS, UMR 7039), Université de Lorraine, BP 239, \\ 54506 Vandouvre-lès-Nancy, France \\ cedric.join@univ-lorraine.fr \\ ${ }^{c} S P E$ (CNRS, UMR 6134), Università di Corsica Pasquale Paoli, 20250 Corte, France \\ voyant@univ-corse.fr \\ ${ }^{d}$ AL.I.E.N. (ALgèbre pour Identification 8 Estimation Numériques), \\ 7 rue Maurice Barrès, 54330 Vézelise, France. \\ \{michel.fliess, cedric.join\}@alien-sas.com \\ ${ }^{e}$ Projet Non-A, INRIA Lille - Nord-Europe, France \\ ${ }^{f}$ Hôpital de Castelluccio, Unité de Radiothérapie, BP 85, 20177 Ajaccio, France
}

\begin{abstract}
Short-term forecasts and risk management for photovoltaic energy is studied via a new standpoint on time series: a result published by P. Cartier and Y. Perrin in 1995 permits, without any probabilistic and/or statistical assumption, an additive decomposition of a time series into its mean, or trend, and quick fluctuations around it. The forecasts are achieved by applying quite new estimation techniques and some extrapolation procedures where the classic concept of "seasonalities" is fundamental. The quick fluctuations allow to define easily prediction bands around the mean. Several convincing computer simulations via real data, where the Gaussian probability distribution law is not satisfied, are provided and discussed. The concrete implementation of our setting needs neither tedious machine learning nor large historical data, contrarily to many other viewpoints.
\end{abstract}

Keywords: Solar energy, short-term forecasts, prediction bands, time series, mean, quick fluctuations, persistence, risk, volatility, normality tests.

\footnotetext{
${ }^{*}$ Corresponding author.
} 


\section{Introduction}

2 Many scientific works and technological issues (see, e.g., Hagenmeyer et al. 3 (2016) ) are related to the Energiewende, i.e., the internationally known German 4 word for the "transition to renewable energies." Among them weather prediction 5 is crucial. Its history is a classic topic (see, e.g., Lynch (2008) and references therein). Reikard (2009) provides an excellent introduction to our more specific subject, i.e., short-term forecasting: "The increasing use of solar power as a source of electricity has led to increased interest in forecasting radiation over short time horizons. Short-term forecasts are needed for operational planning, switching sources, programming backup, and short-term power purchases, as well as for planning for reserve usage, and peak load matching." Time series analysis (see, e.g., Antonanzas et al. (2016)) is quite popular for investigating such situations: See, e.g., Bacher et al. (2009); Behrang et al. (2010); Boland (1997, 2008, 2015a b); Diagne et al. (2013); Duchon et al. (2012); Fortuna et 15 al. (2016); Grantham et al. (2016); Hirata et al. (2017); Inman et al. (2013); 16 Lauret et al. (2015); Martín et al. (2010); Ordiano et al. (2016); Paoli et al. 17 (2010); Prema et al. (2015); Reikard (2009); Trapero et al. (2015); Voyant et al. (2011, 2013, 2015); Wu et al. (2011); Yang et al. (2015); Zhang et al. (2015), ...,

and references therein. The developed viewpoints are ranging from the rather classic setting, stemming from econometrics to various techniques from artificial intelligence and machine learning, like artificial neural networks.

No approach will ever rigorously produce accurate predictions, even nowcasting, i.e., short-term forecasting. To the best of our knowledge, this unavoidable uncertainty, which ought to play a crucial rôle in the risk management of solar energy, starts only to be investigated (see, e.g., David et al. (2016); Ordiano et al. (2016); Rana et al. (2015, 2016); Scolari et al. (2016); Trapero (2016)). As noticed by some authors (see, e.g., David et al. (2016); Trapero (2016), this lack of precision might be related to volatility, i.e., a most popular word in econometrics and financial engineering. Let us stress however the following criticisms, that are borrowed from the financial engineering literature: 
1. Wilmott (2006) (chap. 49, p. 813) writes: Quite frankly, we do not know what volatility currently is, never mind what it may be in the future.

2. According to Mandelbrot et al. (2004), the existing mathematical definitions suffer from poor probabilistic assumptions.

3. Goldstein et al. (2007) exhibits therefore multiple ways for computing volatility which are by no means equivalent and might even be contradictory and therefore misleading.

A recent conference announcement (Join et al. (2016)) is developed here. It is based on a new approach to time series that has been introduced for financial engineering purposes (Fliess et al. (2009, 2011, 2015a b)). A theorem due to Cartier et al. (1995) yields under very weak assumptions on time series an additive decomposition into its mean, or trend, and quick fluctuations around it. Let us emphasize the following points:

- The probabilistic/statistical nature of those fluctuations does not play any rôle!

- No modeling via difference/differential equations is necessary: it is a model-free setting ${ }^{2}$

- Implementation is possible without arduous machine learning and large historical data.

A clear-cut definition of volatility is moreover provided. It is inspired by the

\footnotetext{
${ }^{1}$ This fact should be viewed as fortunate since this nature is rather mysterious if real data are involved.

${ }^{2}$ At least two other wordings, namely "nonparametric" or "data-driven," instead of "modelfree" would have been also possible. The first one however is almost exclusively related to the popular field of nonparametric statistics (see, e.g., Härdle et al. (2004); Wasserman (2006)), that has been also encountered for photovoltaic systems (see, e.g., Ordiano et al. (2016)). The second one has also been recently used, but in a different setting (see, e.g., Ordiano et al. (2017)). Let us highlight the numerous accomplishments of model-free control (Fliess et al. (2013)) in engineering. See for instance renewable energy Bara et al. (2017), Jama et al. (2015), Join et al. (2010), and agricultural greenhouses Lafont et al. (2015).
} 
mean absolute error (MAE) which has been proved already to be more convenient in climatic and environmental studies than the root mean square error (RMSE) (Willmott et al. (2005)). This fact is to a large extent confirmed by Chai et al. (2014) by Section 3.2, which shows that the fluctuations are not Gaussian. See, e.g., (Hyndman (2006)) for further theoretical investigations. Confidence intervals, i.e., a well known notion in statistics (Cox et al. (1974); Willink (2013)), do not make much sense since the probabilistic nature of the uncertainty is unknown. We are therefore replacing them by prediction bands ${ }^{3}$ They mimic to some extent the Bollinger bands (Bollinger (2001)) from technical analysis, i.e., a widespread approach to financial engineering (see, e.g., Béchu et al. (2014); Kirkpatrick et al. (2010)). To pinpoint the efficiency of our tools, numerical experiments via real data stemming from two sites are presented.

Our paper is organized as follows. Time series are the core of Section 2 . where algebraic nowcasting and prediction bands are respectively presented in Sections 2.4 and 2.7. The numerical experiments are presented and discussed in Section 3. Considerations on future investigations are presented in Section 4

\section{Time series}

\subsection{Nonstandard analysis: A short introduction}

Robinson (1996) introduced nonstandard analysis in the early 60's (see, e.g., Dauben (1995)). It is based on mathematical logic and vindicates Leibniz's ideas on "infinitely small" and "infinitely large" numbers. Its presentation by Nelson (1977) (see also Nelson (1987) and Diener et al. (1995, 1989)), where the logical background is less demanding, has become more widely used. As demonstrated by Harthong (1981), Lobry (2008), Lobry et al. (2008), and several other authors, nonstandard analysis is a marvelous tool for clarifying in a most intuitive way various questions from applied sciences.

\footnotetext{
${ }^{3}$ We might also employ the terminology confidence bands. To the best of our knowledge, it has been already employed elsewhere but with another definitions (see, e.g., Härdle et al. (2004)).
} 
78 2.2.1. A nonstandard definition of time series

Take a time interval $[0,1]$. Introduce as often in nonstandard analysis the infinitesimal sampling

$$
\mathfrak{T}=\left\{0=t_{0}<t_{1}<\cdots<t_{\nu}=1\right\}
$$

79 where $t_{i+1}-t_{i}, 0 \leq i<\nu$, is infinitesimal, i.e., "very small." A time series $X$ 80 is a function $\mathfrak{T} \rightarrow \mathbb{R}$.

\subsubsection{The Cartier-Perrin theorem}

The Lebesgue measure on $\mathfrak{T}$ is the function $\ell$ defined on $\mathfrak{T} \backslash\{1\}$ by $\ell\left(t_{i}\right)=$ $t_{i+1}-t_{i}$. The measure of any interval $[c, d] \subset \mathfrak{T}, c \leq d$, is its length $d-c$. The integral over $[c, d]$ of the time series $X(t)$ is the sum

$$
\int_{[c, d]} X d \tau=\sum_{t \in[c, d]} X(t) \ell(t)
$$

\footnotetext{
${ }^{4} a \simeq b$ means that $a-b$ is infinitesimal.

${ }^{5}$ The set $R$ is said to be rare (Cartier et al. (1995)) if, for any standard real number $\alpha>0$, there exists an internal set $A \supset R$ such that $m(A) \leq \alpha$.
} 
A time series $\mathcal{X}: \mathfrak{T} \rightarrow \mathbb{R}$ is said to be quickly fluctuating, or oscillating, if, and only if, it is $S$-integrable and $\int_{A} \mathcal{X} d \tau$ is infinitesimal for any quadrable subset 6

Let $X: \mathfrak{T} \rightarrow \mathbb{R}$ be a $S$-integrable time series. Then, according to the Cartier-Perrin theorem (Cartier et al. $(1995))$. 7 the additive decomposition

$$
X(t)=E(X)(t)+X_{\text {fluctuat }}(t)
$$

holds where

- $E(X)(t)$, which is called the mean, or trend $\left.\right|^{8}$ is Lebesgue integrable;

- $X_{\text {fluctuat }}(t)$ is quickly fluctuating.

The decomposition (2) is unique up to an additive infinitesimal quantity. Let us stress once again that the above mean is independent of any probabilistic modeling $]^{9}$

\subsection{Volatility}

According to

- our discussion about mean absolute errors (MAE) in Section 1 .

- the fact, which follows at once from the Cartier-Perrin theorem, that $\mid X-$ $E(X) \mid$ is $S$-integrable,

define the volatility $\operatorname{vol}(X)(t)$ of $X(t)$ by

$$
\operatorname{vol}(X)(t)=E(|X-E(X)|)(t)
$$

$E(|X-E(X)|)(t)$ in Equation (3) is nothing else than the mean of $\mid X(t)-$ $E(X)(t) \mid$.

\footnotetext{
${ }^{6} \mathrm{~A}$ set is quadrable Cartier et al. $(1995)$ if its boundary is rare.

${ }^{7}$ The presentation in the article by Lobry et al. 2008) is less technical. We highly recommend it. Note that it also includes a fruitful discussion on nonstandard analysis.

8 "Trend" would be the usual terminology in technical analysis (see, e.g., Béchu et al. (2014); Kirkpatrick et al. (2010). It was therefore used by Fliess et al. (2009).

${ }^{9}$ Let us mention that Cartier et al. (1995) also introduced the notion of martingales (see, e.g., Williams (1991)) without using any probabilistic tool.
} 


\subsubsection{First calculations}

Start with a polynomial time function

$$
p_{1}(t)=a_{0}+a_{1} t, \quad t \geq 0, \quad a_{0}, a_{1} \in \mathbb{R},
$$

of degree 1. Rewrite thanks to classic operational calculus (see, e.g., Yosida (1984) $)^{11} p_{1}$ as

$$
P_{1}=\frac{a_{0}}{s}+\frac{a_{1}}{s^{2}}
$$

Multiply both sides by $s^{2}$ :

$$
s^{2} P_{1}=a_{0} s+a_{1}
$$

Take the derivative of both sides with respect to $s$, which corresponds in the time domain to the multiplication by $-t$ :

$$
s^{2} \frac{d P_{1}}{d s}+2 s P_{1}=a_{0}
$$

The coefficients $a_{0}, a_{1}$ are obtained via the triangular system of equations (4)5. 5e get rid of the time derivatives, i.e., of $s P_{1}, s^{2} P_{1}$, and $s^{2} \frac{d P_{1}}{d s}$, by multiplying both sides of Equations (4)-(5) by $s^{-n}$, i.e., $n \geq 3$ (resp $n \geq 2$ ) for Equation (4) (resp. (5)). The corresponding iterated time integrals are lowpass filters (see, e.g., Shenoi (2006)): they attenuate the corrupting noises, which are viewed as highly fluctuating phenomena $($ Fliess $(2006))$. A quite short time

\footnotetext{
${ }^{10}$ Those techniques have already been successfully employed in engineering. In signal processing, see, e.g., the recent publications by Beltran-Carbajala et al. (2017) and Morales et al. (2016).

11 The computations below are often presented via the classic Laplace transform (see, e.g., Doetsch (1976)). Then $s$ is called the Laplace variable.
} 
window $[0, \mathfrak{t}]$ is sufficient for obtaining accurate estimates $\hat{a}_{0}, \hat{a}_{1}$, of $a_{0}, a_{1}$, where $n=2,3$ :

$$
\hat{a}_{0}=\frac{2}{\mathfrak{t}^{2}} \int_{0}^{\mathfrak{t}}(2 \mathfrak{t}-3 \tau) p(\tau) d \tau
$$

and

$$
\hat{a}_{1}=-\frac{6}{\mathfrak{t}^{3}} \int_{0}^{\mathfrak{t}}(\mathfrak{t}-2 \tau) p(\tau) d \tau
$$

This last formula shows that a derivative estimate is obtained via integrals. Lanczos (1956) was perhaps the first author to suggest such an approach. In practice, the above integrals are of course replaced by straightforward linear digital filters (see, e.g., Shenoi (2006)).

\subsection{Back to time series and short-term forecasts}

Assume that the following rather weak assumption holds true: the mean $E(X(t))$ may be associated with a differentiable real-valued time function. Then, on a short time lapse, $E(X(t))$ is well approximated by a polynomial function of degree 1. The above calculations yield via sliding time windows numerical estimates $E(X)_{\text {estim }}(t)$ and $\frac{d}{d t} E(X)_{\text {estim }}(t)$ of the mean and its derivative. Causality is taken into account via backward calculations with respect to time. As in (Fliess et al. $(2009,2011))$, forecasting the time series $X(t)$ boils down to an extrapolation of its mean $E(X)(t)$. If $T>0$ is not "too large," i.e., a few minutes in our context, a first order Taylor expansion yields the following extrapolation for prediction at time $t+T$

$$
X_{\text {predict }}(t+T)=E(X)_{\text {estim }}(t)+\left(\frac{d}{d t} E(X)_{\text {estim }}(t)\right) \times T
$$

\subsection{Forecasting for a larger time horizon}

With forecasts for a time horizon equal to 1 hour, Equation (6) would provide poor results. Seasonalities, i.e., a more or less periodic pattern, which is classic in time series analysis (see, e.g., Brockwell et al. (1991); Mélard (2008)) will be used here. A single day is an obvious season with respect to photovoltaic energy. Figures 7, 10 show that the corresponding pattern may be reasonably well approximated by a parabola $D(t)=\alpha_{2} t^{2}+\alpha_{1} t+\alpha_{0}$. Standard least 
square techniques permit to obtain such a suitable parabola, that is the set of parameters $\left\{\alpha_{0}, \alpha_{1}, \alpha_{2}\right\}$, only with the data collected during a single day. Replace Equation (6) by

$$
X_{\text {predict }}(t+T)=E(X)_{\text {estim }}(t)+(\dot{D}(t-1 . \text { day })) \times T
$$

where

- $T>0$ is the time horizon, here between 30 minutes and 1 hour;

- $D(t-1$.day $)$ is estimated via the data from the day before;

- $\dot{D}(t-1$.day $)$ is its derivative.

This formula is useful since the parabola is erasing the bumps and the hollows on the trend. Taking derivatives around such bumps and holes leads obviously to a wrong forecasting for a larger time horizon.

\subsection{Prediction bands}

Equation (3) yields the prediction $\operatorname{Vol}_{\text {predict }}(X)(t+T)$ of the volatility at time $t+T$ via the following persistence law (Lauret et al. (2015)):

$$
\operatorname{Vol}_{\text {predict }}(X)(t+T)=\operatorname{Vol}(X)(t)=E(|X-E(X)|)(t)
$$

Define via Equation (8) the first prediction band

$$
\begin{gathered}
\begin{array}{c}
\mathrm{CB}_{1}(t+T) \\
=
\end{array} \begin{array}{c}
\text { predict }(t+T)-\mathrm{Vol}_{\text {predict }}(X)(t+T) \\
\leq \mathrm{CB}_{1}(t+T) \leq
\end{array} \\
X_{\text {predict }}(t+T)+\operatorname{Vol}_{\text {predict }}(X)(t+T)=\overline{\mathrm{CB}_{1}(t+T)}
\end{gathered}
$$

In order to improve it, set

$$
\begin{gathered}
\frac{\mathrm{CB}_{2}(t+T)}{2}=X_{\text {predict }}(t+T)-\alpha_{t+T} \operatorname{Vol}_{\text {predict }}(X)(t+T) \\
\leq \mathrm{CB}_{2}(t+T) \leq \\
X_{\text {predict }}(t+T)+\alpha_{t+T} \operatorname{Vol}_{\text {predict }}(X)(t+T)=\overline{\mathrm{CB}_{2}(t+T)}
\end{gathered}
$$


Diffuse radiation $I_{d, c l s k}$ is defined by

$$
I_{d, c l s k}=I_{g, c l s k}-I_{b, c l s k}
$$

\footnotetext{
${ }^{12}$ The quantity $68 \%$ is obviously inspired by the theory confidence intervals with respect to Gaussian probability distributions.
} 
It yields

$$
\begin{aligned}
& \mathrm{CB}_{3}(t+T)=\max \left(I_{d, c l s k}(t), X_{\text {predict }}(t+T)-\alpha_{t+T} \operatorname{Vol}_{\text {predict }}(X)(t+T)\right) \\
& \leq \mathrm{CB}_{3}(t+T) \leq \\
& \min \left(1.1 \times I_{g, c l s k}(t), X_{\text {predict }}(t+T)+\alpha_{t+T} \operatorname{Vol}_{\text {predict }}(X)(t+T)\right)=\overline{\mathrm{CB}_{3}(t+T)}
\end{aligned}
$$

where $\min (\square(t), \triangle(t))$ and $\max (\square(t), \triangle(t))$ are respectively the minimum and maximum values of the arguments $\square(t)$ and $\triangle(t)$ at time $t$.

The safety margin corresponding to the multiplicative factor 1.1 takes into account a modeling error on $I_{g, c l s k}(\operatorname{Ineichen}(2008))$, whereas $I_{d, c l s k}$ does not necessitate such a correction (Ineichen (2008)).

\section{Computer experiments via real data}

Three time horizons are considered: 1, 15 and 60 minutes. The following points should be added:

- no exogenous variable,

- no need of large historical data,

- unsupervised method.

\subsection{Data}

The full year data were collected from two sites in 2013 by means of CMP11 pyranometer (Kipp \& Zonen):

- Nancy in the East of France. It has usually a relatively narrow annual temperature range.

- Ajaccio in Corsica, a French island in the Mediterranean sea. This coastal town has hot and sunny summers and mild winters. 


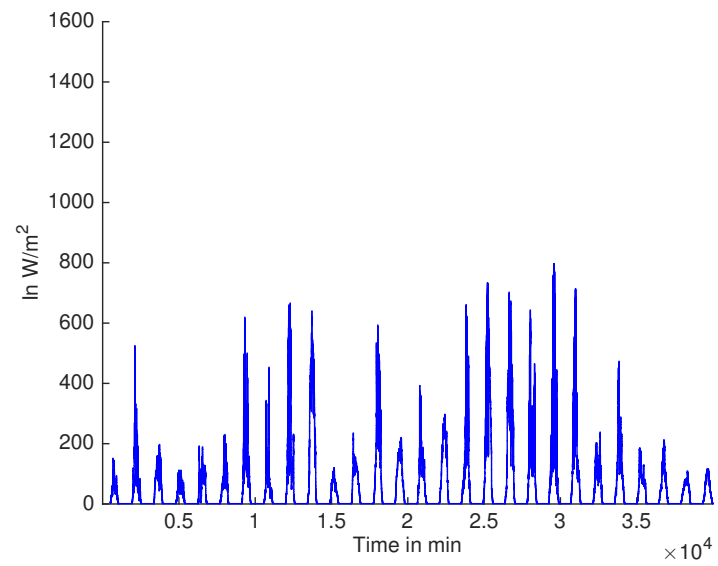

(a) Nancy

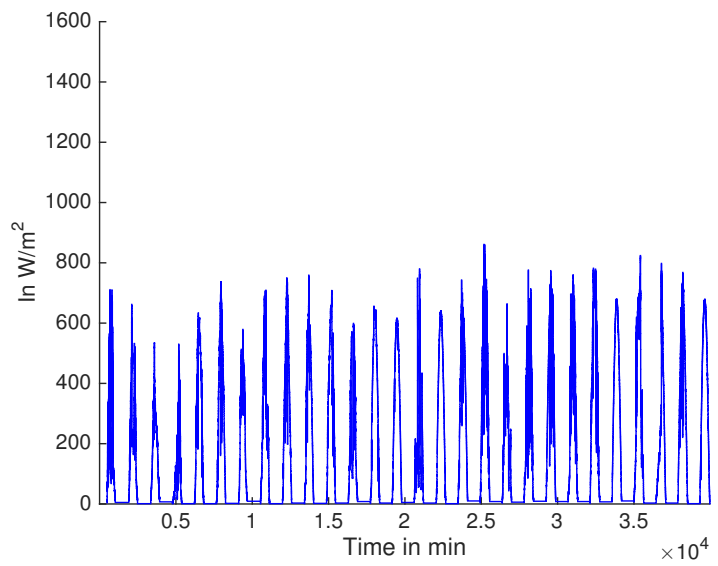

(b) Ajaccio

Figure 1: Global irradiation profile for the two sites in February

The time granularity of our solar irradiance measurements is 1 minute. Missing values for the sites are less than $2 \% 13$ See Figures 1 and 2 for excerpts. The numerical values of the parameters in Equations 11 and 12 , are ${ }^{14}$

- Nancy: $\tau_{g}=0.49, g=0.39, \tau_{b}=0.66, b=0.51$;

- Ajaccio: $\tau_{g}=0.43, g=0.33, \tau_{b}=0.64, b=0.51$.

\subsection{Normality tests}

To better justify our definitions of volatility in Section 2.3 and of prediction bands in Section 2.7. we show that, if the fluctuations around the trends are viewed as random variables, they are not Gaussian. Three classic tests (see, e.g., Jarque et al. (1987); Judge et al. (1988); Thode (2002)) are used:

- Jarque-Bera,

- Kolmogorov-Smirnov,

\footnotetext{
${ }^{13}$ The data are cleaned as in David et al. (2016).

${ }^{14}$ See, e.g., the WEB site of AERONE'T Data Synergy Tool.
} 


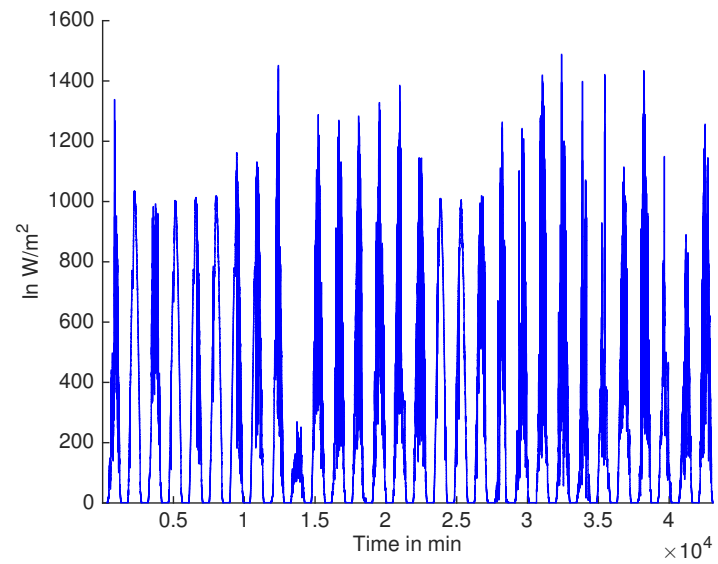

(a) Nancy

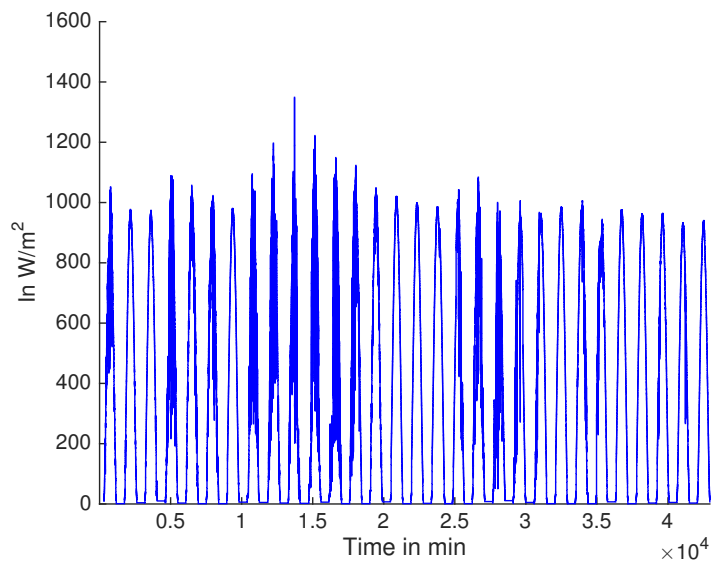

(b) Ajaccio

Figure 2: Global irradiation profile for the two sites in June

- Lilliefors.

Figures 3, 4, 5 and 6, show most clearly that the Gaussian property is not satisfied.

\subsection{Presentation of some results}

Figures 1 and 2 present global irradiation during 1 month. Figure 1 displays an irregular radiation behavior during winter. As shown by Figure 1 - (a) this is especially true for Nancy. During summer, Figure 2 exhibits a nice daily seasonality even if some deteriorations show up for Nancy.

The red line in Figures 7, 8, 10, 11, on the one hand, and in Figures 9, 12 on the other hand, show the forecasts according respectively to Equations (6) and (7).

Remark 3.1. We follow a common practice by removing night hours, i.e., hours where the solar elevation $h$ in Equations $11-(12$ is less than 10 degrees. 


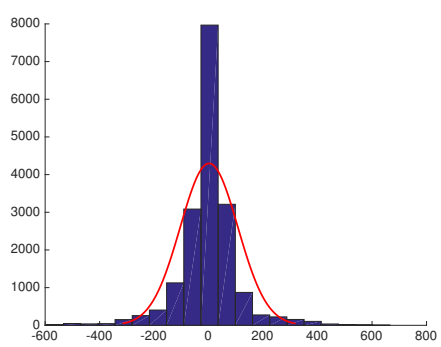

(a) $5 \mathrm{~min}$

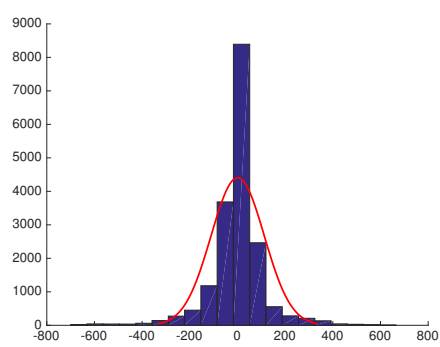

(b) $15 \mathrm{~min}$

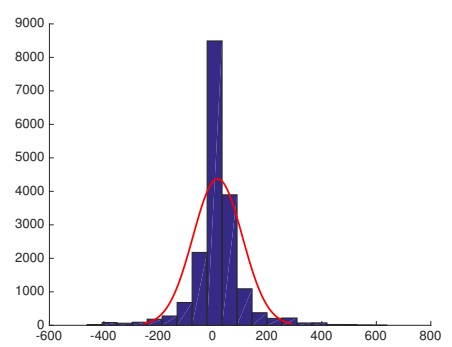

(c) $60 \mathrm{~min}$

Figure 3: Nancy, February : Signal distribution (blue) and the Gaussian distribution (red)

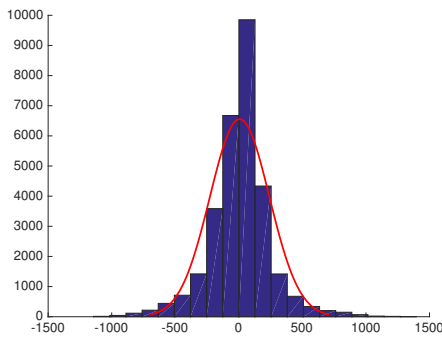

(a) $5 \mathrm{~min}$

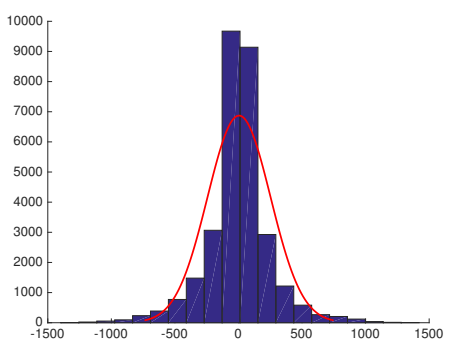

(b) $15 \mathrm{~min}$

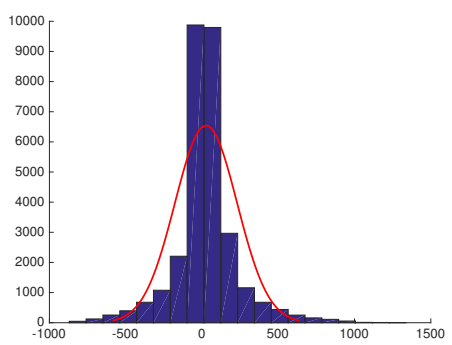

(c) $60 \mathrm{~min}$

Figure 4: Nancy, June : Signal distribution (blue) and the Gaussian distribution (red)

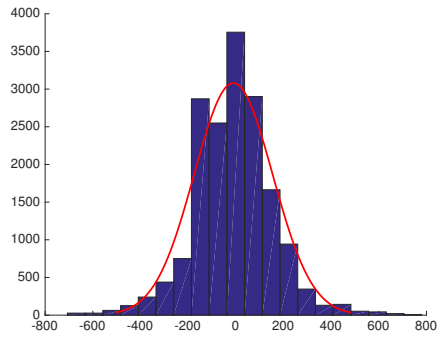

(a) $5 \mathrm{~min}$

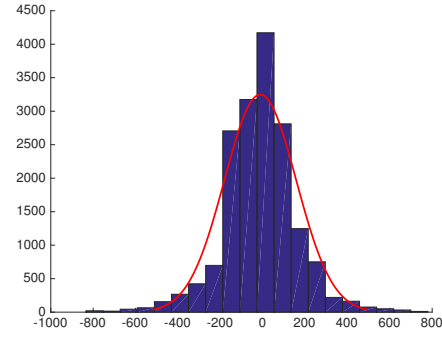

(b) $15 \mathrm{~min}$

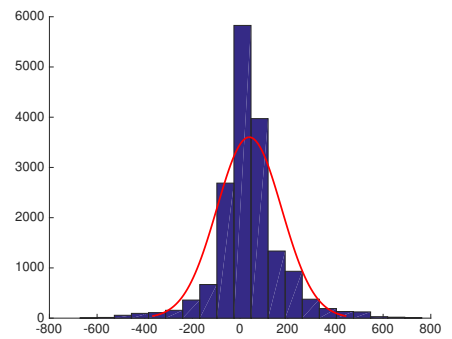

(c) $60 \mathrm{~min}$

Figure 5: Ajaccio, February : Signal distribution (blue) and the Gaussian distribution (red) 


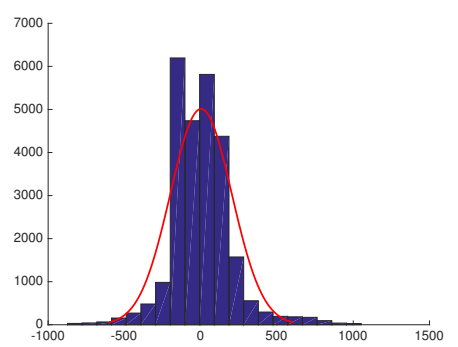

(a) $5 \mathrm{~min}$

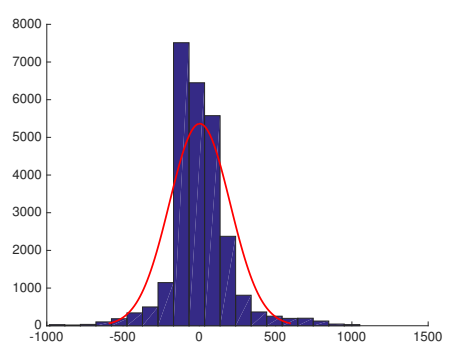

(b) $15 \mathrm{~min}$

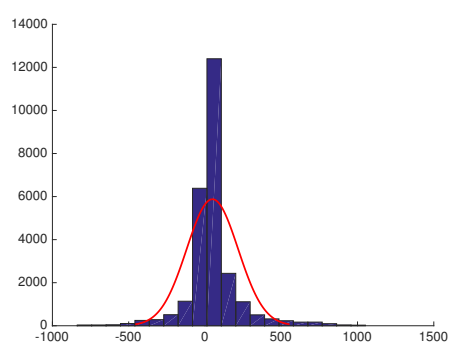

(c) $60 \mathrm{~min}$

Figure 6: Ajaccio, June : Signal distribution (blue) and the Gaussian distribution (red)

The prediction bands defined in Section 2.7 are also displayed in the previous figures. Note the following points:

1. by construction, $C B_{2}$ yields larger bands than $C B_{1}$,

2. the mean interval length is reduced with $C B_{3}$,

3. the widths of the bands increase with the time horizon,

4. Daily profiles, Figures 7, 8, 9 on the one hand, and 10, 11, 12 on the other hand demonstrate that forecasts are better in June than in February.

In order to quantify comparisons, introduce the following quantities:

- The Mean Interval Length, or MIL stems from the Mean Relative Error (MRL) (Rana et al. (2015, 2016); Scolari et al. (2016)). It is given by

$$
\mathrm{MIL}_{i}=\frac{\sum_{k=1}^{N} \mathrm{BW}_{i}\left(t_{k}\right)}{\sum_{k=1}^{N} X\left(t_{k}\right)} \quad i=1,2,3
$$

where

$-N$ is the number of measurements,

- $\mathrm{BW}_{i}=\overline{C B_{i}}\left(t_{k}+T\right)-\underline{C B_{i}}\left(t_{k}+T\right) \geq 0$, is the band width,

- $X\left(t_{k}\right) \geq 0$ the irradiance measurement.

- The Prediction Interval Coverage Probability, or PICP, (Rana et al. (2015. 2016); Scolari et al. (2016)) is defined by

$$
\mathrm{PICP}_{i}=\frac{\sum_{k=1}^{M} c_{k}}{M} \quad i=1,2,3
$$


where

- $M$ is the number of predictions,

$-c_{k}=1$ if the prediction is inside the bands, i.e., $\underline{C B_{i}}\left(t_{k}+T\right) \leq$ $X\left(t_{k}+T\right) \leq \overline{C B_{i}}\left(t_{k}+T\right)$,

$-c_{k}=0$ otherwise.

A quite large $\mathrm{MIL}_{i}$ with a $\mathrm{PICP}_{i}$ close to 1 is inefficient for grid management. Our objective is a large $\mathrm{PICP}_{i}$ and a low $\mathrm{MIL}_{i}$. Consequently, a compromise is required. Figures 13, 14,15 and 16 present $\mathrm{MIL}_{i}$ vs $\mathrm{PICP}_{i}$ for all forecasting horizons.

On these Figures, four areas characterise the CB qualities. Thus, if a bound is in the "good" area, the result is more interesting than in the "bad" and even more than in the "very bad" areas but less interesting than in the "very good" zone. According to the clear sky concept and to the ad-hoc computing methodologies Mueller et al. (2004); Ineichen (2008), the measured global irradiance is between the computed irradiance under clear sky and under totally cloudy sky. So $100 \%$ of the predictions, i.e., $\mathrm{PICP}_{i}=1$, should be included between the bounds defined by the global radiation $I_{g, c l s k}$ and the diffuse radiation $I_{d, c l s k}$. Uncertainties and Solis modeling errors explain why it is not always the case.

The space is divided in four zones. The blue line is the vertical limit. It corresponds to a PICP of 0.5 : it means that $50 \%$ of predictions are in the band. The green line is the horizontal limit. It defines the limit of relevance: all the intervals with a $M I L>1$ are not really interesting since the bands are too large. For case $1\left(C B_{1}\right)$ and $2\left(C B_{2}\right)$, we find coherent results because the PICP are respectively close to $70 \%$ and $50 \%$. Regarding case 2, the MIL is too important. Regarding case $3\left(C_{3}\right)$, the best compromise between low MIL and high PICP is obtained thanks to the clear sky model.

\subsection{Some preliminary comments on comparisons}

Comparing our results with the huge set of numerical calculations in the whole academic literature is obviously beyond the reach of a single journal pub- 


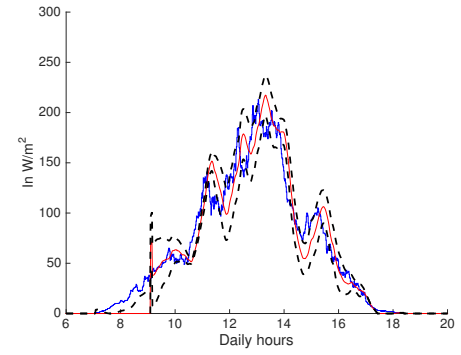

(a) $C B_{1}$

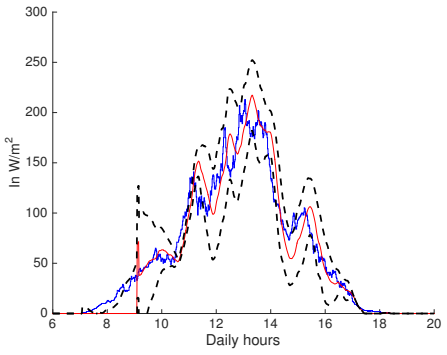

(b) $C B_{2}$

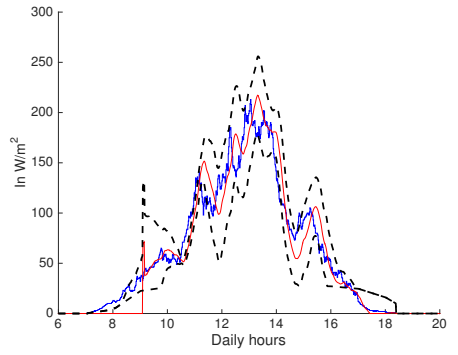

(c) $C B_{3}$

Figure 7: Nancy, February, 5min forecasting: irradiance (blue), its prediction (red) and prediction band (black - -)

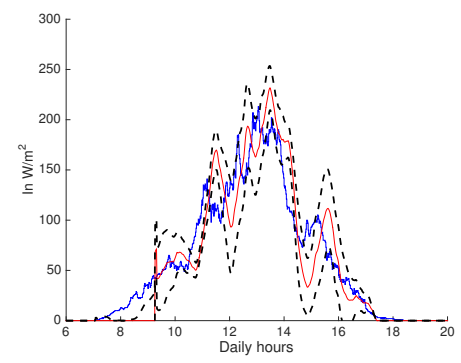

(a) $C B_{1}$

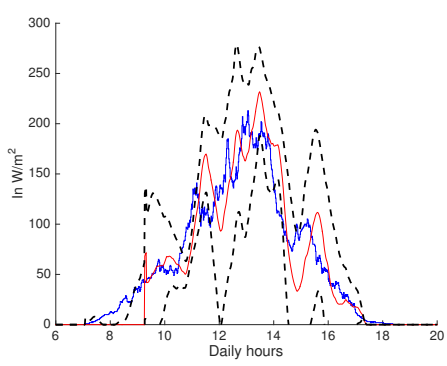

(b) $C B_{2}$

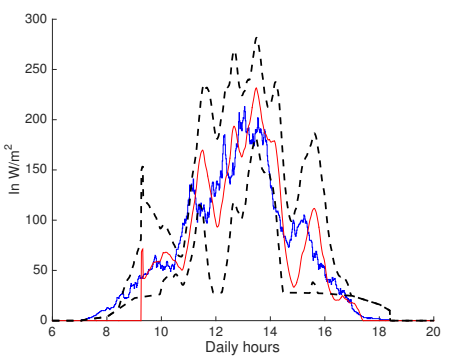

(c) $C B_{3}$

Figure 8: Nancy, February, 15min forecasting: irradiance (blue), its prediction (red) and prediction band (black - -) 


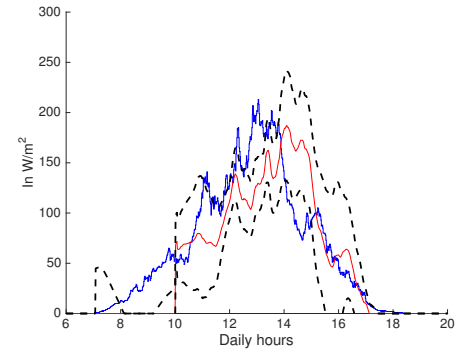

(a) $C B_{1}$

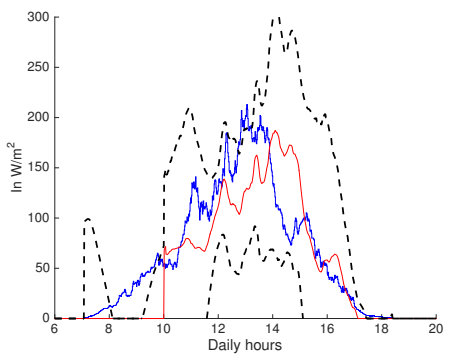

(b) $C B_{2}$

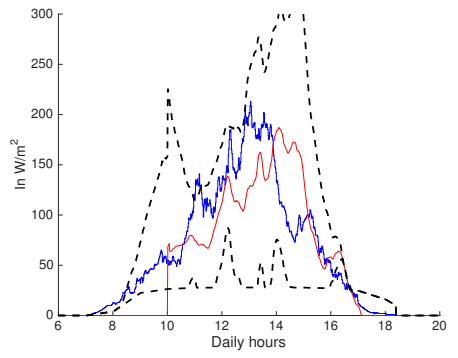

(c) $C B_{3}$

Figure 9: Nancy, February, 60min forecasting: irradiance (blue), its prediction (red) and prediction band (black - -)

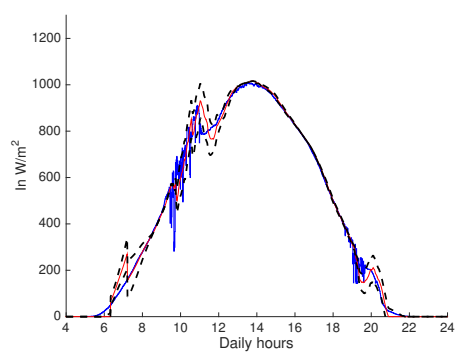

(a) $C B_{1}$

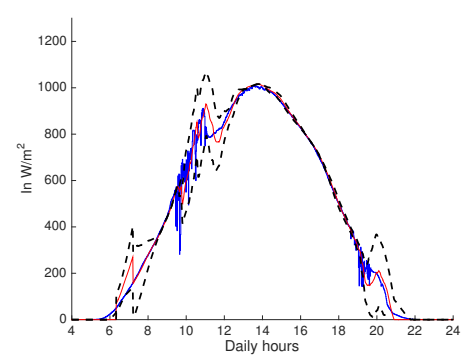

(b) $C B_{2}$

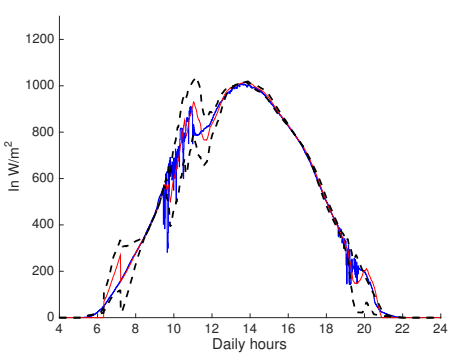

(c) $C B_{3}$

Figure 10: Nancy, June, 5min forecasting: irradiance (blue), its prediction (red) and prediction band (black - -) 


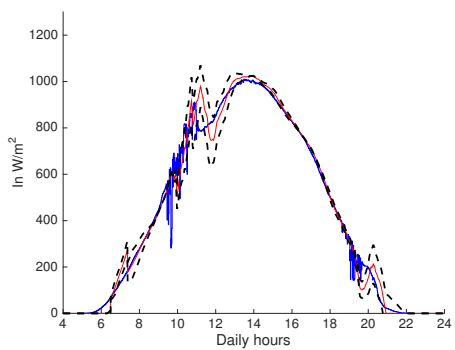

(a) $C B_{1}$

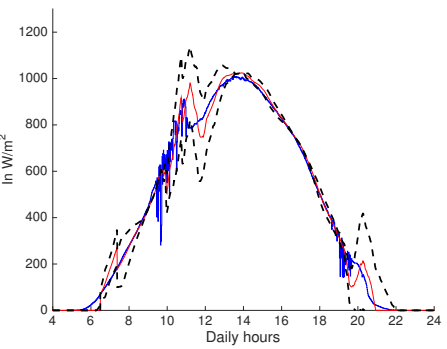

(b) $C B_{2}$

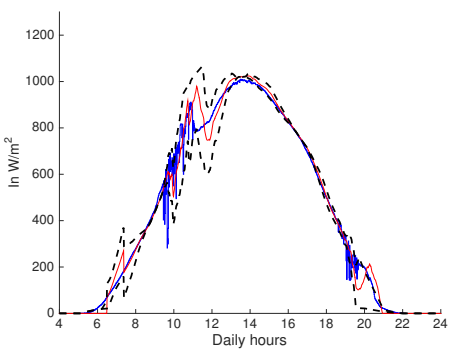

(c) $C B_{3}$

Figure 11: Nancy, June, 15min forecasting: irradiance (blue), its prediction (red) and prediction band (black - -)

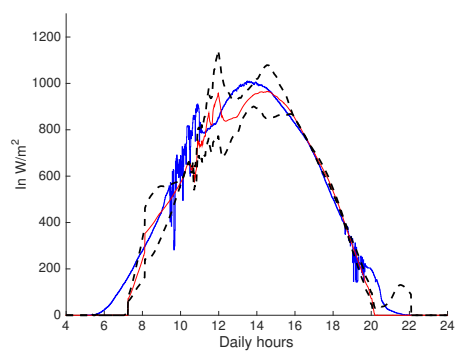

(a) $C B_{1}$

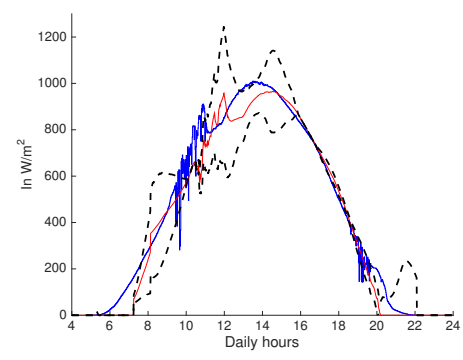

(b) $C B_{2}$

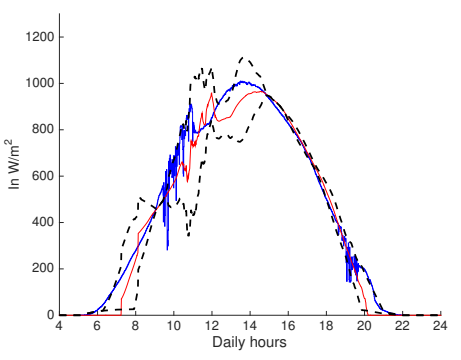

(c) $C B_{3}$

Figure 12: Nancy, June, 60min forecasting: irradiance (blue), its prediction (red) and prediction band (black - -)

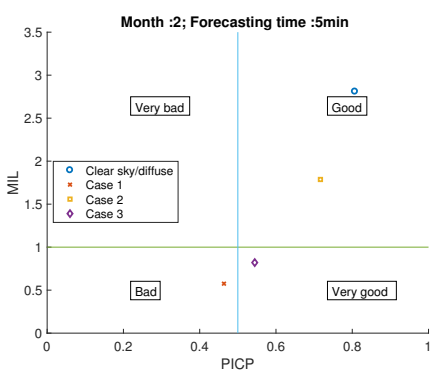

(a) $5 \mathrm{~min}$

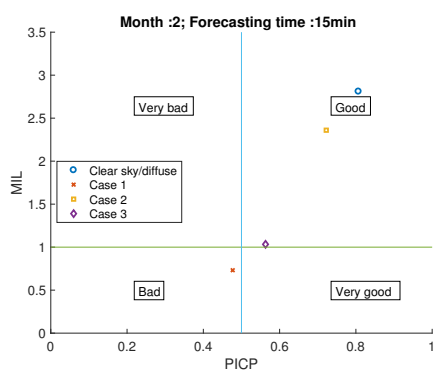

(b) $15 \mathrm{~min}$

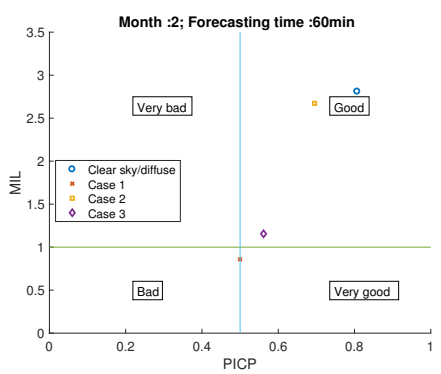

(c) $60 \mathrm{~min}$

Figure 13: Nancy, February: Performance evaluation 


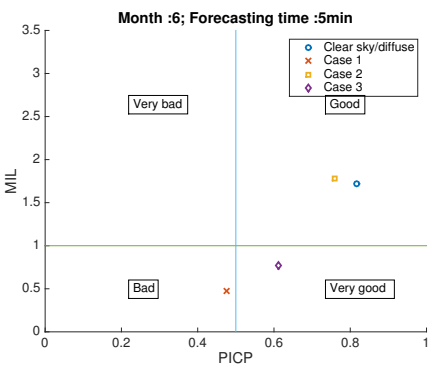

(a) $5 \mathrm{~min}$

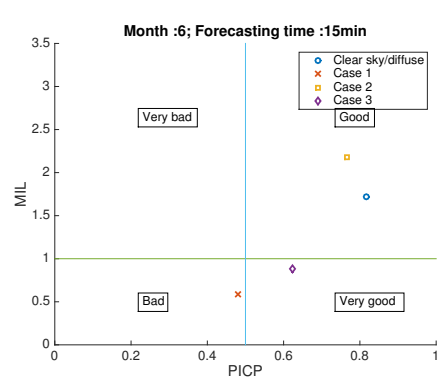

(b) $15 \mathrm{~min}$

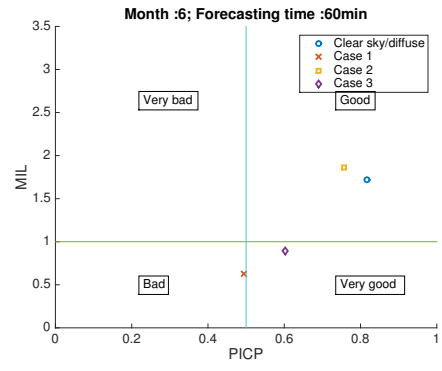

(c) $60 \mathrm{~min}$

Figure 14: Nancy, June: Performance evaluation

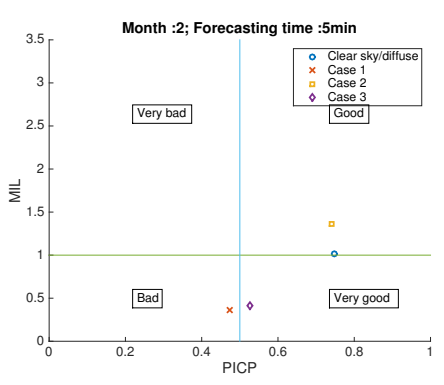

(a) $5 \mathrm{~min}$

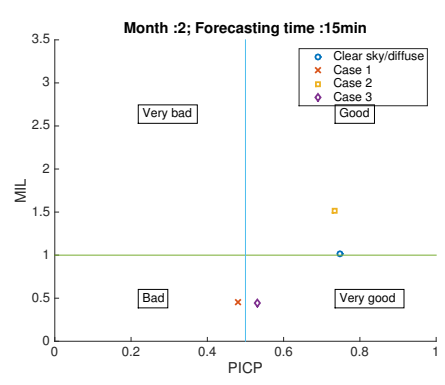

(b) $15 \mathrm{~min}$

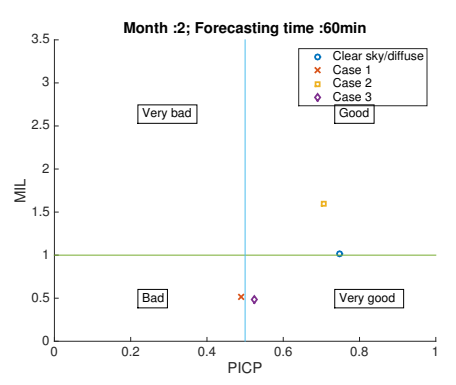

(c) $60 \mathrm{~min}$

Figure 15: Ajaccio, February: Performance evaluation

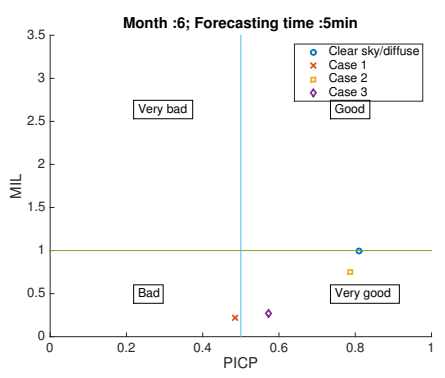

(a) $5 \mathrm{~min}$

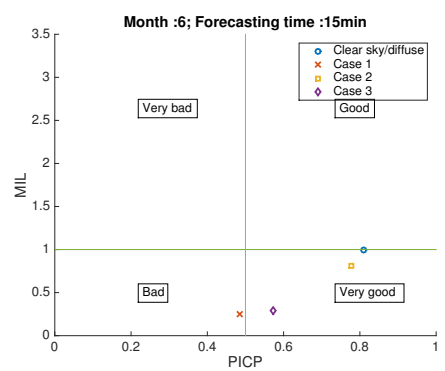

(b) $15 \mathrm{~min}$

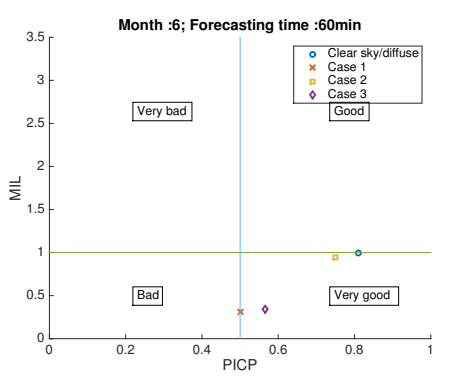

(c) $60 \mathrm{~min}$

Figure 16: Ajaccio, June: Performance evaluation 
lication. Let us nevertheless summarize the observations in Join et al. (2014); Voyant et al. (2015) on short-term forecasting with respect to artificial neural networks:

1. the performances of the "algebraic" setting, that is presented here, are perhaps slightly better than those via neural nets. For the irradiance (resp. irradiation), the mean absolute error (MAE) between the forecasts and the true values is $26.6 \%$ (resp. $23.9 \%$ ) vs $35.44 \%$ (resp. $22.36 \%$ ).

2. When looking at big data and machine learning, the behavior of the algebraic setting looks much better. Neural nets need data during 3 years whereas the algebraic viewpoint only 1 day.

WWith respect, for instance, to Grantham et al. (2016); David et al. (2016); Trapero (2016), note that time-consuming and cumbersome calibrations to obtain a convincing probability law, a time series modeling via a difference equation, and a suitable autoregressive conditional heteroskedasticity $(A R C H)$ or generalized autoregressive conditional heteroskedasticity $(G A R C H){ }^{15}$ become quite irrelevant.

\section{Conclusion}

The positive results obtained in this paper ought of course to be verified by considering more diverse situations and by launching more thorough comparisons than in Section 3.4. For future researches, let us emphasize the two following directions:

- The possibility of extending our techniques to larger time horizons is another key point.

- Asymmetric prediction bands might be useful in practice for energy management.

\footnotetext{
${ }^{15}$ The popular concepts of ARCH and GARCH were respectively introduced by Engle (1982) and Bollerslev (1986). Everyone should read the harsh comments by Mandelbrot et al. $(2004)$.
} 
- Is the causality analysis by Fliess et al. (2015a) useful to improve our forecasting techniques if other facts are taken into account (see, e.g., Badosa et al. (2015))?

The concrete implementation of our approach should be rather straightforward.

Finally, if our standpoint encounters some success, the probabilistic techniques (see, e.g., Appino et al. (2018); Gneiting et al. (2014); Hong et al. (2016); Lauret et al. (2017)), which are a today mainstay in all the fields of energy forecasting, price included, might become clearly less central.

Acknowledgements. The authors thank warmly the anonymous reviewers for their most helpful comments.

\section{References}

Antonanzas, J., Osorio, N., Escobar, R., Urraca, R., Martinez-de-Pison, F.J., Antonanzas-Torres, F., 2016. Review of photovoltaic power forecasting. Solar Ener. 136, 78-111.

Appino, R.R., Ordiano, J.Á.G., Mikut, R., Faulwasser, T., Hagenmeyer, V., 2018. On the use of probabilistic forecasts in scheduling of renewable energy sources coupled to storages. Appl. Ener. 210, 1207-1218.

Bacher, P., Madsen, H., Nielsen, H.A., 2009. Online short-term solar power forecasting. Solar Ener. 83, 1772-1783.

Badosa, J., Haeffelin, M., Kalecinski, N., Bonnardot, F., Jumaux, G., 2015. Reliability of day-ahead solar irradiance forecasts on Reunion Island depending on synoptic wind and humidity conditions. Solar Ener. 115, 315-321.

Bara, O., Olama, M., Djouadi, S., Kuruganti, T., Fliess, M., Join, C., 2017. Model-free load control for high penetration of solar photovoltaic generation. 49th North Amer. Power Symp., Morgantown. 
Behrang, M.A., Assareh, E., Ghanbarzadeh, A., Noghrehabadi, A.R., 2010. The potential of different artificial neural network (ANN) techniques in daily global solar radiation modeling based on meteorological data, Solar Ener. 84, 1468-1480.

Béchu , T., Bertrand, É., Nebenzahl, J., 2014. L'analyse technique (7 $7^{e}$ éd.). Economica.

Beltran-Carbajala, F., Silva-Navarro, G., 2017. A fast parametric estimation approach of signals with multiple frequency harmonics, Elec. Power Syst. Res. 144, 157-162.

Boland, J., 1997. Time series analysis of climatic variables. Solar Ener. 55, 377-388.

Boland, J., 2008. Time series modelling of solar radiation. In Badescu, V. (Ed.): Modeling Solar Radiation at the Earth's Surface: Recent Advances, Springer, 283-326.

Boland, J., 2015a. Spatial-temporal forecasting of solar radiation. Renew. Ener. $75,607-616$.

Boland, J., 2015b. Additive versus multiplicative seasonality in solar radiation time series. 21st Int. Congr. Model. Simu., Gold Coast.

Bollerslev, T., 1986. Generalized autoregressive conditional heteroskedasticity, J. Economet. 31, 307-327.

Bollinger, J., 2001. Bollinger on Bollinger Bands. McGraw-Hill.

Brockwell, P.J., Davis, R.A., 1995. Time Series: Theory and Methods, (2nd ed.). Springer.

Cartier, P., Perrin, Y., 1995. Integration over finite sets, in F. \& M. Diener, Eds: Nonstandard Analysis in Practice, Springer, 195-204. 
Chai, T., Draxler, R.R., 2014. Root mean square error (RMSE) or mean absolute error (MAE)? - Arguments against avoiding RMSE in the literature. Geosci. Model Dev. 7, 1247-1250.

Cox, D.R., Hinkley, D.V., 1974. Theoretical Statistics. Chapman \& Hall.

Cros, S., Liandrat ,O., Sébastien, N., Schmutz, N., Voyant, C., 2013. Clear sky models assessment for an operational PV production forecasting solution. 28th Europ. Photovolt. Solar Energy Conf. Exhib., Villepinte.

Dauben, J.W., 1995. Abraham Robinson - Nonstandard Analysis: A Personal and Mathematical Odyssey. Princeton University Press.

David, M., Ramahatana, F., Trombe, P.J., Lauret, P., 2016. Probabilistic forecasting of the solar irradiance with recursive ARMA and GARCH models. Solar Ener. 133, 55-72.

Diagne, M., David, M., Lauret, P., Boland, J., Schmutz, N., 2013. Review of solar irradiance forecasting methods and a proposition for small-scale insular grids. Renew. Sustain. Energy Rev. 27, 65-76.

Diener, F., Diener, M., 2013. Tutorial. In F. \& M. Diener (Eds): Nonstandard Analysis in Practice, Springer, 1-21.

Diener, F., Reeb, G., 1989. Analyse non standard, Hermann.

Doetsch, G., 1976. Einführung in Theorie und Anwendung der LaplaceTransformation (3. Auflage), Springer.

Duchon, C., Hale, R., 2012. Time Series Analysis in Meteorology and Climatology: An Introduction. Wiley-Blackwell.

Engle, R.F., 1982. Autoregressive conditional heteroscedasticity with estimates of the variance of United Kingdom Inflation. Econometrica 50, 987-1007.

Fliess, M., 2006. Analyse non standard du bruit. C.R. Acad. Sci. Paris 342, $797-802$. 
Fliess, M., Join, C., 2009. A mathematical proof of the existence of trends in financial time series. In A. El Jai, L. Afifi, E. Zerrik (Eds): Systems Theory: Modeling, Analysis and Control, Presses Universitaires de Perpignan, 43-62.

Fliess, M., Join, C., 2013. Model-free control, Int. J. Contr. 86, 2228-2252.

Fliess, M., Join, C., 2015a. Towards a new viewpoint on causality for time series. ESAIM ProcS 49, 37-52.

Fliess, M., Join, C., 2015b. Seasonalities and cycles in time series: A fresh look with computer experiments. Paris Finan. Manag. Conf., Paris.

Fliess, M., Join, C., Hatt, F., 2011. A-t-on vraiment besoin d'un modèle probabiliste en ingénierie financière ? Conf. Médit. Ingén. Sûre Syst. Compl., Agadir.

Fliess, M., Join, C., Sira-Ramírez, H., 2008a. Non-linear estimation is easy. Int. J. Model. Identif. Contr. 4, 12-27.

Fliess, M., Sira-Ramírez, H., 2003. An algebraic framework for linear identification. ESAIM Contr. Optimiz. Calc. Variat. 9, 151-168.

Fliess, M., Sira-Ramírez, H., 2008b. Closed-loop parametric identification for continuous-time linear systems via new algebraic techniques. H. Garnier \& L. Wang (Eds): Identification of Continuous-time Models from Sampled Data, Springer, 362-391.

Fortuna, L., Nunnari, G., Nunnari, S., 2016. Nonlinear Modeling of Solar Radiation and Wind Speed Time Series. Springer.

Gneiting, T., Katzfuss, M., 2014. Probabilistic forecasting. Ann. Rev. Stat. Appl. 1, 125-151.

Goldstein, D.G., Taleb, N.N., 2007. We don’t quite know what we are talking about when we talk about volatility. J. Portfolio Manage. 33, 84-86. 
Grantham, A., Gel, Y.R., Boland, J., 2016. Nonparametric short-term probabilistic forecasting for solar radiation. Solar Ener. 133, 465-475.

Hagenmeyer, V., Çakmak, H.K., Düpmeier, C., Faulwasser, T., Isele, J., Keller, H.B., Kohlhepp, P., Kühnapfel, U., Stucky, U., Waczowicz, S., Mikut, R., 2016. Information and communication technology in energy lab 2.0: Smart energies system simulation and control center with an open-street-map-based power flow simulation example. Energy Technol. 4, 145-162.

Härdle,W., Müller, M., Sperlich, S., Werwatz, A., 2004. Nonparametric and Semiparametric Models. Springer.

Harthong, J., 1981. Le moiré. Adv. Appl. Math. 2, 21-75.

Hirata, Y., Aihara, K., 2017. Improving time series prediction of solar irradiance after sunrise: Comparison among three methods for time series prediction. Solar Ener. 149, 294-301.

Hong, T., Pinson, P., Fan, S., Zareipour, H., Troccoli, A., Hyndman, R. J., 2016. Probabilistic energy forecasting: Global energy forecasting competition 2014 and beyond. Int. J. Forecast. 32, 896-913.

Hyndman, R.J., 2006. Another look at forecast accuracy metrics for intermittent demand. Int. J.Applied Forecast., 4, 43-46.

Ineichen, P., 2008. A broadband simplified version of the Solis clear sky model. Solar Ener. 82, 758-762.

Inman, R.H., Pedro, H.T.C., Coimbra, C.F.M., 2013. Solar forecasting methods for renewable energy integration. Progr. Ener. Combust. Sci. 47, 2479-2490.

Jama, M.A., Noura, H., Wahyudie, A., Assi, A, 2015. Enhancing the performance of heaving wave energy converters using model-free control approach. Renew. Energy 83, 931-941.

Jarque, C.M., Bera, A.K., 1987. A test for normality of observations and regression residuals. Int. Stat. Rev, 55, 163-172. 
Join, C., Voyant, C., Fliess, M., Muselli, M., Nivet, M.-L., Paoli, C., Chaxel, F., 2014. Short-term solar irradiance and irradiation forecasts via different time series techniques: A preliminary study. 3rd Int. Symp. Environ. Friendly Energy Appl., Paris.

Join, C., Fliess, M., Voyant, C., Chaxel, F., 2016. Solar energy production: Short-term forecasting and risk management. 8th IFAC Conf. Manufact. Model. Manage. Contr., Troyes.

Join, C., Robert, G., Fliess, M., 2016. Vers une commande sans modèle pour aménagements hydroélectriques en cascade. $6^{e}$ Conf. Internat. Francoph. Automat., Nancy.

Judge, G.G., Griffiths, W.E., Hill, R.C., Lütkepohl, H., Lee, T.-C.,1988. Introduction to the Theory and Practice of Econometrics (2nd ed.), Wiley.

Kirkpatrick II, C.D., Dahlquist, J.A., 2010. Technical Analysis: The Complete Resource for Financial Market Technicians (2nd ed.). FT Press.

Lafont, F., Balmat, J.-F., Pessel, N., Fliess, M., 2015. A model-free control strategy for an experimental greenhouse with an application to fault accommodation. Comput. Electron. Agricult. 110, 139-149.

Lanczos, C., 1956. Applied Analysis. Prentice Hall.

Lauret, P., David M., Pedro H.T.C., 2017. Probabilistic solar forecasting using quantile regression models. Energies 10, 1591, doi:10.3390/en10101591

Lauret, P., Voyant, C., Soubdhan, T., David, M., Poggi, P., 2015. A benchmarking of machine learning techniques for solar radiation forecasting in an insular context. Solar Ener. 112, 446-457.

Lobry, C., 2008. La méthode des élucidations successives. ARIMA 9, 171-193.

Lobry, C., Sari, T., 2008. Nonstandard analysis and representation of reality. Int. J. Contr. 39, 535-576. 
Lynch, P., 2008. The origins of computer weather prediction and climate modeling. J. Comput. Phys. 227, 3431-3444.

Mandelbrot, N., Hudson, R.L., 2004. The (Mis)Behavior of Markets: A Fractal View of Risk, Ruin, and Reward. Basic Books.

Martín, L., Zarzalejo, L.F., Polo, J., Navarro, A., Marchante, R., Cony, M., 2010. Prediction of global solar irradiance based on time series analysis: Application to solar thermal power plants energy production planning. Solar Ener. 84, 1772-1781.

Mboup, M., Join, C., Fliess, M., 2009. Numerical differentiation with annihilators in noisy environment. Numer. Algor. 50, 439-467.

Mélard, G., 2009. Méthodes de prévision à court terme. Ellipses - Presses Universitaires de Bruxelles.

Morales, R., Segura, E., Somolinos, J.A., Núñez, L.R., Sira-Ramírez, H., 2016. Online signal filtering based on the algebraic method and its experimental validation. Mechan. Syst. Signal Process. 66-67, 374-387.

Mueller, R.W., Dagestad, K.F., Ineichen, P., Schroedter-Homscheidt, M., Cros, S., Dumortier, D., Kuhlemann, R., Olseth, J.A., Piernavieja, G., Reise, C., Wald, L., Heinemann, D., 2004. Rethinking satellite-based solar irradiance modelling: The SOLIS clear-sky module. Remote Sensing Environm. 91, $160-174$.

Nelson, E., 1977. Internal set theory. Bull. Amer. Math. Soc. 83, 1165-1198.

Nelson, E., 1987. Radically Elementary Probability Theory. Princeton University Press.

Ordiano, J.À.G., Doneit, W., Waczowicz, S., Gröll, L., Mikut, R., Hagenmeyer, V., 2016. Nearest-neighbor based non-parametric probabilistic forecasting with applications in photovoltaic systems. Proc. 26. Workshop Comput. Intel., Dortmund. 
Ordiano, J.À.G., Waczowicz, S., Reischl, M., Mikut, R., Hagenmeyer, V., 2017. Photovoltaic power forecasting using simple data-driven models without weather data. Comput. Sci. Res. Develop. 32, 237-246.

Paoli, C., Voyant, C., Muselli, M., Nivet, M.-L., 2010. Forecasting of preprocessed daily solar radiation time series using neural networks. Solar Ener. 84 $2146-2160$.

Prema, J.L., Rao, K.U., 2015. Development of statistical time series models for solar power prediction. Renew. Ener. 83, 100-109.

Rana, M., Koprinska, I., Agelidis, V.G., 2015. 2D-interval forecasts for solar power production. Solar Ener. 122, 191-203.

Rana, M., Koprinska, I., 2016. Neural Network Ensemble Based Approach for 2D-Interval Prediction of Solar Photovoltaic Power. Energies 9, 829-846.

Reikard, G., 2009. Predicting solar radiation at high resolutions: A comparison of time series forecasts. Solar Ener., 83, 342-349.

Robinson, A., 1996. Non-standard Analysis (revised ed.). Princeton University Press.

Scolari, E., Torregrossa, D., Le Boudec, J.-Y., Paolone, M., 2016. Ultra-shortterm prediction intervals of photovoltaic AC active power. Int. Conf. Proba. Meth. Appl. Power Syst., Beijing.

Shenoi, B.A., 2006. Introduction to Digital Signal Processing and Filter Design, Wiley.

Sira-Ramírez, H., García-Rodríguez, C., Cortès-Romero, J., Luviano-Juárez, A., 2013. Algebraic Identification and Estimation Methods in Feedback Control Systems, Wiley.

Thode, H.C., 2002. Testing for Normality, Marcel Dekker. 
Trapero, J.R., 2016. Calculation of solar irradiation prediction intervals combining volatility and kernel density estimates. Energy 114, 266-274.

Trapero, J.R., Kourentzes, N., Martin, A., 2015. Short-term solar irradiation forecasting based on Dynamic Harmonic Regression. Energy 84, 289-295.

Voyant, C., Join, C., Fliess, M., Nivet M.-L., Muselli, M., Paoli, C., 2015. On meteorological forecasts for energy management and large historical data: A first look. Renewable Energy Power Qualit. J. 13, ISSN 2172-038 X

Voyant, C., Muselli, M., Paoli, C., Nivet, M.-L., 2011. Optimization of an artificial neural network dedicated to the multivariate forecasting of daily global radiation. Energy 36, 348-359.

Voyant, C., Paoli, C., Muselli, M., Nivet, M.-L., 2013. Multi-horizon solar radiation forecasting for Mediterranean locations using time series models. Renew. Sustain. Energy Rev. 28, 44-52.

Voyant, C., Soubdhan, T., Lauret, P., David, M., Muselli, M., 2015. Statistical parameters as a means to a priori assess the accuracy of solar forecasting models. Energy 90, 671-679.

Wasserman, L., 2006. All of Nonparametric Statistics. Springer.

Williams, D., 1991. Probability with Martingales. Cambridge University Press. Willink, R., 2013. Measurement Uncertainty and Probability. Cambridge University Press.

Wilmott, P, 2006. Paul Wilmott on Quantitative Finance, 3 vol. (2nd ed.). .

Willmott, CJ., Matsuura, K., 2005. Advantages of the mean absolute error (MAE) over the root mean square error (RMSE) in assessing average model performance. Climate Res. 30, 79-82.

Wu, J., Chan, C.K., 2011. Prediction of hourly solar radiation using a novel hybrid model of ARMA and TDNN. Solar Ener. 85, 111-119. 
491 Yang, D., Sharma, V., Ye, Z., Lim, L.I., Zhao, L., Aryaputer, A.W., 2015.

492 Forecasting of global horizontal irradiance by exponential smoothing, using decompositions. Energy 85, 808-817.

Yosida, K., 1984. Operational Calculus (translated from the Japanese). Springer.

Zhang, J., Florita, A., Hodge, B.-M., Lu, S., Banunarayanan, V., Brockway, A.M., 2015. A suite of metrics for assessing the performance of solar power forecasting. Solar Ener. 111, 157-175. 